\title{
Outcomes after endoscopic mucosal resection or esophagectomy for submucosal esophageal adenocarcinoma
}

\author{
David B. Nelson, MD, ${ }^{\mathrm{a}}$ Rajeev Dhupar, MD, ${ }^{\mathrm{b}}$ Riham Katkhuda, BS, ${ }^{\mathrm{c}}$ Arlene Correa, $\mathrm{PhD},{ }^{\mathrm{a}}$
}

Alexei Goltsov, PhD, ${ }^{a}$ Dipen Maru, MD ${ }^{c}$ Boris Sepesi, MD, ${ }^{a}$ Mara B. Antonoff, MD, ${ }^{a}$

Reza J. Mehran, MD, ${ }^{\mathrm{a}}$ David C. Rice, MD, ${ }^{\mathrm{a}}$ Ara A. Vaporciyan, MD, ${ }^{\mathrm{a}}$ Marta Davila, MD, ${ }^{\mathrm{d}}$

Raquel Davila, MD, ${ }^{\mathrm{d}}$ Sonia Betancourt, MD, Jaffer Ajani, MD, ${ }^{\mathrm{f}}$ and Wayne L. Hofstetter, MD, FACS ${ }^{\mathrm{a}}$

\section{ABSTRACT}

Objectives: Endoscopic mucosal resection (EMR) is a diagnostic and potentially therapeutic option for patients with submucosal esophageal adenocarcinoma. However, there are significant concerns regarding the risk of lymph node metastasis. Our purpose was to construct a comparative effectiveness analysis comparing recurrence patterns after therapeutic EMR or esophagectomy.

Methods: Patients who underwent therapeutic EMR or esophagectomy from 2007 to 2015 with pathologically staged submucosal adenocarcinoma were identified from a departmental database. Cancer-related outcomes were compared among an unmatched as well as a propensity matched cohort. Risk stratification was also used to compare results among those with a low, medium, or high risk of nodal metastasis.

Results: Seventy-two patients met criteria for analysis, among whom 23 underwent therapeutic EMR with esophageal preservation and 49 underwent esophagectomy. Median follow-up was 43 months. Patients who underwent esophagectomy had larger, deeper tumors. Esophageal preservation was associated with an increased risk of local recurrence $(P=.01)$, but not distant recurrence $(P=.44)$. After propensity matching, there continued to be no difference in distant recurrence rate $(P=.66)$. In a risk-stratified analysis, low-risk patients showed no recurrences or cancer-related deaths, however, high-risk patients showed a trend toward increased distant recurrence after therapeutic EMR.

Conclusions: Esophageal preservation after therapeutic EMR was associated with an increased risk of local recurrence. Among low-risk patients, either strategy resulted in excellent cancer control. However, among high-risk patients, esophageal preservation showed a trend toward increased distant failure. These findings should prompt further investigation to determine optimal treatment for patients with submucosal esophageal adenocarcinoma. (J Thorac Cardiovasc Surg 2018;156:406-13)

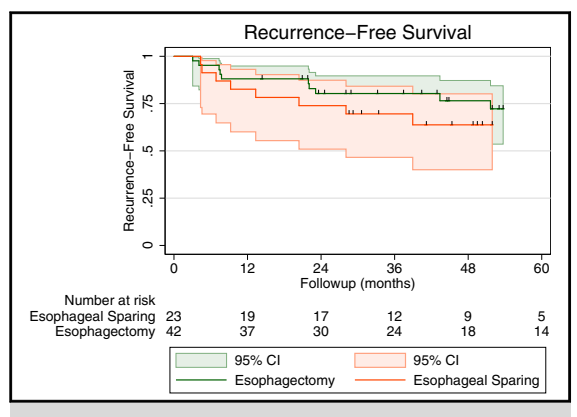

Recurrence-free survival. EMR shown in orange; surgery in green.

\section{Central Message}

Therapeutic EMR for submucosal esophageal adenocarcinoma is associated with an increased risk of local recurrence. Low-risk patients had excellent outcomes regardless of treatment modality.

\section{Perspective}

The use of endoscopic resection for submucosal esophageal cancer is increasing, however, evidence is limited to case series or small retrospective analyses. We show that EMR is associated with a risk of local recurrence and thus close surveillance is required. Most local recurrences able to be salvaged. EMR might have a therapeutic role among carefully selected, low-risk submucosal esophageal cancer patients.

See Editorial Commentaries pages 414 and 416.

See Editorial page 404.
From the Departments of ${ }^{\mathrm{a}}$ Thoracic and Cardiovascular Surgery, ${ }^{\mathrm{c}}$ Pathology, ${ }^{\mathrm{d}} \mathrm{Gas}-$ troenterology, ${ }^{\mathrm{e}}$ Diagnostic Imaging, and ${ }^{\mathrm{f}}$ Gastrointestinal Medical Oncology, The University of Texas MD Anderson Cancer Center, Houston, Tex; and ${ }^{\mathrm{b}}$ Department of Cardiothoracic Surgery, University of Pittsburgh, Pittsburgh, Pa.

This study was funded by departmental support.

Read at the 97th Annual Meeting of The American Association for Thoracic Surgery, Boston, Massachusetts, April 29-May 3, 2017.

Received for publication May 22, 2017; revisions received Jan 2, 2018; accepted for publication Feb 1, 2018; available ahead of print March 28, 2017.

\footnotetext{
Address for reprints: Wayne L. Hofstetter, MD, FACS, Department of Thoracic and Cardiovascular Surgery, The University of Texas MD Anderson Cancer Center, 1515 Holcombe Blvd, Houston, TX 77030 (E-mail: whofstetter@mdanderson. org).

$0022-5223 / \$ 36.00$

Copyright (C) 2018 by The American Association for Thoracic Surgery

https://doi.org/10.1016/j.jtcvs.2018.02.093
} 


\section{Abbreviations and Acronyms}

$\mathrm{CI}=$ confidence interval

$\mathrm{EMR}=$ endoscopic mucosal resection

EUS $=$ endoscopic ultrasound

LVI = lymphovascular invasion

$\mathrm{SM}=$ submucosal layer

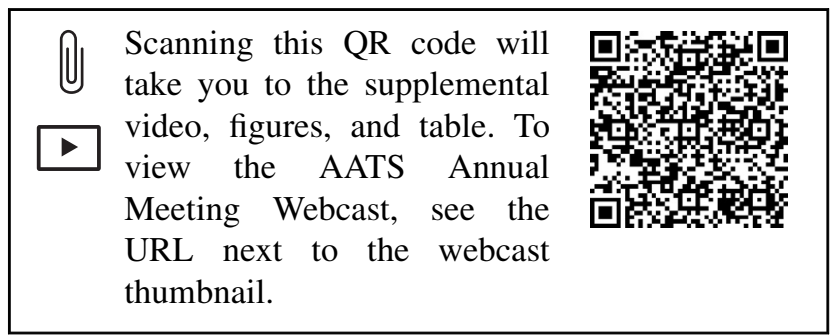

Esophageal cancer is the seventh most common cause of cancer deaths in the United States, with an estimated 15,590 deaths in 2015 alone. ${ }^{1}$ Traditionally, surgery has been the preferred treatment for localized esophageal cancer, however, in recent years therapeutic endoscopic mucosal resection (EMR) has been increasingly used. Esophageal cancer that is confined to the mucosal layer can be readily cleared using EMR. ${ }^{2-5}$ However, when esophageal cancer reaches the submucosal layer (SM), the risk of lymph node metastasis substantially increases. ${ }^{6}$ The SM of the esophagus is rich with lymphatic networks that can lead to rapid dissemination. Poor differentiation, presence of lymphovascular invasion (LVI), depth of invasion, and tumor diameter $\geq 2 \mathrm{~cm}$ have all been implicated to be risk factors for lymph node metastasis for esophageal cancer that is within the SM. ${ }^{6-12}$ On the contrary, patients who lack these aggressive features could conversely have a low risk of lymph node metastasis and be managed successfully with EMR.

Often, EMR is used as a diagnostic tool to improve staging before surgery (Video 1). EMR can either be followed by an esophagectomy, or can be considered therapeutic with no further surgical procedure. Therefore, all EMR procedures can be broadly categorized as diagnostic or therapeutic on the basis of intent. Some centers have been advocating for endoscopic therapy among carefully selected, low-risk submucosal esophageal cancer patients in the hope of avoiding the morbidity and mortality of esophagectomy. ${ }^{13}$ Endoscopic therapy for submucosal esophageal cancer has drastically increased, with approximately $20.9 \%$ of submucosal esophageal cancer being treated definitively with endoscopic resection in 2010, up from $6.6 \%$ in 2004. ${ }^{14}$ Although therapeutic EMR is rapidly expanding,

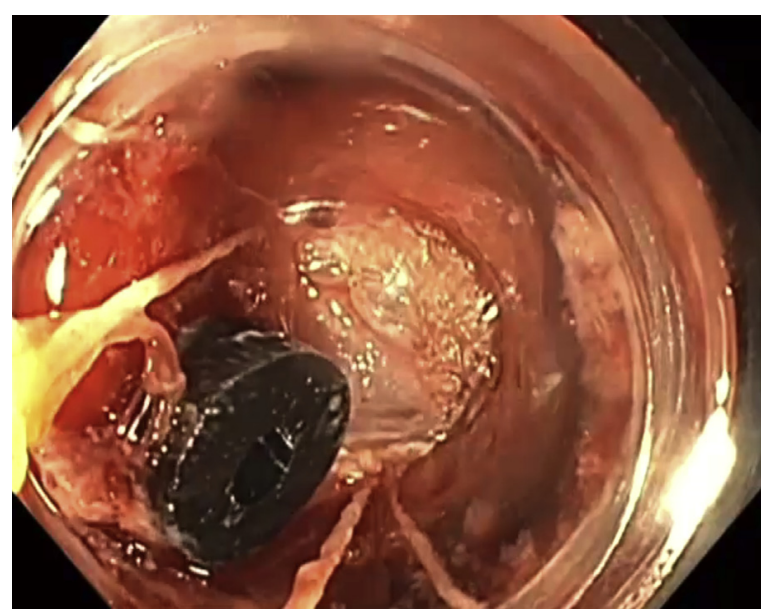

VIDEO 1. Endoscopic mucosal resection. Video available at: http://www. jtcvsonline.org/article/S0022-5223(18)30730-X/fulltext.

much of our understanding of its efficacy is derived from theoretical risk models predicting the risk of lymph node metastases using anatomic evaluation of esophagectomy specimens. ${ }^{6,8}$ Outcomes research after endoscopic therapy has been lacking. These outcomes are especially crucial to obtain because of the complexity of therapy, including possible adjunctive treatments as well as salvage options for locoregional failures, and because inadequate disease control might result in the unfortunate recurrence of an otherwise curable cancer.

We constructed a comparative effectiveness analysis by comparing the cancer-related outcomes of patients who underwent either therapeutic EMR or esophagectomy for submucosal esophageal adenocarcinoma. We used a risk-stratified analysis with the hypothesis that patients with a low risk of nodal metastasis will fare well regardless of local resection approach, whereas patients with a high risk of nodal metastasis will have improved nodal or distant recurrence after esophagectomy. As a secondary analysis, patients were matched on the basis of their propensity to undergo therapeutic EMR with esophageal preservation.

\section{METHODS}

\section{Patient Selection}

This study was approved by the MD Anderson Cancer Center institutional review board with a waiver of individual informed consent (Protocol PA16-0718; approval date September 3, 2016). Patients who underwent esophagectomy or therapeutic EMR for pathologically confirmed submucosal esophageal adenocarcinoma from 2007 to 2015 were identified from a prospectively maintained departmental database. Patients with a positive deep margin on therapeutic EMR were excluded from analysis, because of the possibility that the depth of invasion was in fact within the muscularis propria or deeper. We excluded patients from either cohort who received pretreatment chemoradiation, had previous treatment for their index esophageal cancer lesion, were medically unfit to receive any further chemoradiation or surgery, or were lost to follow-up. To allow for similar nodal staging workup, we also excluded patients who were not staged with a preprocedure 
endoscopic ultrasound examination. After exclusion of patients with pretreatment chemoradiation, there were no patients with clinically positive regional lymph nodes before treatment (all were $\mathrm{pT} 1 \mathrm{~b}, \mathrm{cN} 0$ ). We permitted esophagectomy patients who did not receive a pre-procedure diagnostic EMR (21 of 42).

\section{Postprocedure Surveillance}

Surveillance was routinely performing using endoscopy as well as positron emission tomography or computed tomography imaging when clinically indicated. Fifteen of 23 patients who underwent definitive EMR as local therapy were subsequently treated with ablative therapy (range, 1-8 treatments). The reasons for no ablative therapy after therapeutic EMR were as follows: 5 received definitive chemoradiation, and 3 were for unknown reasons.

\section{Covariate and Outcome Definition}

Depth of invasion within the SM (1-3) was reported after pathologic review of specimens. For patients with a positive deep margin on the diagnostic EMR who proceeded with esophagectomy, the depth was recorded as SM3. The end points evaluated included local recurrence, regional recurrence, distant recurrence, cancer-related mortality, and recurrence-free survival. Cancer-related mortality was defined as any death while active cancer was present. Local recurrence was defined as any recurrence within the esophagus after therapeutic EMR, including recurrences that were detected after the first post-EMR endoscopy.

\section{Stratified Analysis}

All patients were stratified using a scoring system to assess the risk of nodal metastasis. ${ }^{8}$ Patients were assigned 1 point per $\mathrm{cm}$ of tumor size, 3 points for SM3 invasion, 3 points for poor differentiation, and 6 points for LVI. Low risk is considered 0 to 2 points, medium risk 3 to 5 points, and high risk is $\geq 6$ points.

\section{Statistical Analysis \\ Differences between groups were calculated using Wilcoxon rank sum test, Pearson $\chi^{2}$ test, or Fisher exact test, where appropriate. Observations were censored at the date of last contact. To minimize the effect of lag-time bias against the surgical cohort because of EMR always preceding esophagectomy, initial event time was defined from date of pathologic diagnosis rather than the date of treatment. Any eventual recurrence (either local, regional, or distant) was used for analysis, as opposed to electing to only study isolated recurrences. Kaplan-Meier survival curves were constructed and stratified according to risk of lymph node metastases. The log rank test was used to evaluate for statistical differences between groups. Propensity to undergo therapeutic EMR was estimated using a logistic regression model, taking into account the presence of LVI, depth of invasion, and tumor grade. Patients were matched in a 1:1 fashion using the nearest neighbor method and required a caliper match distance of 0.2 . Covariate balance as well as mirror histograms before and after the match are shown in Table E1 and Figure E1.}

\section{RESULTS \\ Demographic Characteristics}

We identified 42 patients who underwent an esophagectomy and 23 patients who underwent a therapeutic EMR and opted for esophageal preservation (Figure 1). The median follow-up overall was similar between groups: 43.5 months among esophagectomy and 45.1 months among esophageal preservation. Patients who underwent an esophagectomy had larger, deeper tumors than patients who underwent esophageal preservation (Table 1). The rate of LVI and poor differentiation were higher in the

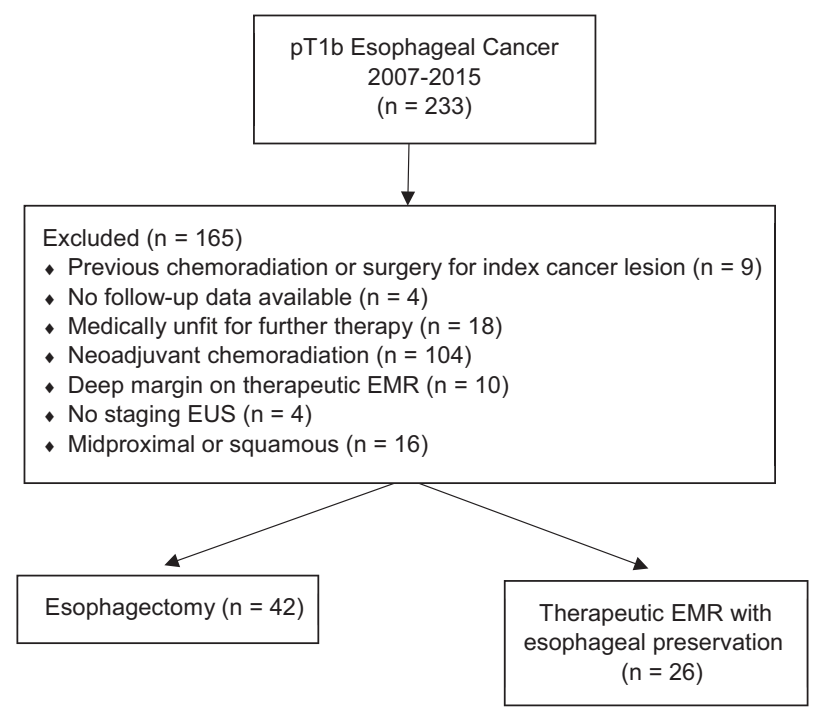

FIGURE 1. Consolidated Standards of Reporting Trials diagram. $E M R$, Endoscopic mucosal resection; EUS, endoscopic ultrasound.

esophagectomy group, but these differences were not statistically significant. Among the 42 esophagectomy patients, $21(50 \%)$ had a previous diagnostic EMR.

\section{Periprocedural Outcomes}

There were no 30-day mortalities among the esophagectomy or esophageal preservation cohorts. Surgical procedures performed were as follows: 27 of 42 patients underwent an Ivor Lewis esophagectomy, 5 of 42 underwent a transhiatal esophagectomy, and 10 of 42 underwent a minimally invasive esophagectomy. After esophagectomy, of the 21 with a previous diagnostic EMR, 11 had no evidence of residual local disease or evidence of regional disease.

Pathologic N1 disease was detected in 7 of 42 surgical patients $(17 \%)$, with no $\mathrm{N} 2$ disease. More patients underwent adjuvant chemoradiation after esophageal preservation ( 6 of $23 ; 26 \%$ ) than esophagectomy ( 2 of 42 ; $5 \% ; P=.012)$.

\section{Recurrence Patterns After Esophageal Preservation}

Local recurrence within 3 years after esophageal preservation was $23.1 \%$ (95\% confidence interval [CI], $10.2 \%-47.2 \%$ ), which represented 5 of 23 patients (Figure 2, A). Patients who developed local recurrence after esophageal preservation underwent repeat EMR ( 2 of 5), surgery ( 1 of 5), or received definitive chemoradiation (2 of 5) despite being offered surgery. Among the 5 patients who had local recurrence, 3 of them did not receive ablative therapy after the EMR. The median time for a local recurrence to occur in the esophageal preservation group was 7 months. There were no regional recurrences among patients who developed local recurrence after esophageal preservation. One patient developed 
TABLE 1. Baseline demographic and tumor characteristics

\begin{tabular}{|c|c|c|c|}
\hline & $\begin{array}{l}\text { Esophageal preservation } \\
\qquad(\mathbf{n}=\mathbf{2 3})\end{array}$ & Esophagectomy $(n=42)$ & $P$ value \\
\hline Median age, $y$ & 71.5 (IQR, 63.8-76.3) & 64.5 (IQR, 61.0-70.0) & .053 \\
\hline Median follow-up, mo & 45.1 & 43.5 & .842 \\
\hline \multicolumn{4}{|l|}{ Sex } \\
\hline Male & $20(87 \%)$ & $36(86 \%)$ & \\
\hline Female & $3(13 \%)$ & $6(14 \%)$ & 1.000 \\
\hline Long Barrett (>3 cm) & $11(48 \%)$ & $17(40 \%)$ & .567 \\
\hline \multicolumn{4}{|l|}{ Depth of invasion } \\
\hline SM1 & $17(74 \%)$ & $18(43 \%)$ & \\
\hline SM2 & $6(26 \%)$ & $5(12 \%)$ & \\
\hline SM3 & 0 & $19(45 \%)$ & .001 \\
\hline \multicolumn{4}{|l|}{ Grade } \\
\hline Well to moderate & $16(70 \%)$ & $20(48 \%)$ & \\
\hline Poor & $7(30 \%)$ & $22(52 \%)$ & .089 \\
\hline \multicolumn{4}{|l|}{ Tumor location } \\
\hline Distal & $8(35 \%)$ & $17(40 \%)$ & \\
\hline GEJ & $15(65 \%)$ & $25(60 \%)$ & .652 \\
\hline Endoscopic tumor size, $\mathrm{cm}$ & 1.5 (IQR, 0.6-2) & 2 (IQR, 1.1-3) & .007 \\
\hline Lymphovascular invasion & $6(26 \%)$ & $20(48 \%)$ & .090 \\
\hline
\end{tabular}

$I Q R$, Interquartile range; $S M$, submucosal layer; $G E J$, gastroesophageal junction.

distant metastasis after definitive chemoradiation and is still undergoing treatment. There were no cancer-related mortalities in this subset of patients.

Regional recurrence within 3 years after esophageal preservation was $4.7 \%$ (95\% CI, $0.7 \%-28.7 \%)$, which represented 1 of 23 patients. This patient presented with concomitant regional and distant supraclavicular nodal involvement. This patient received chemoradiation and subsequently died.

Distant recurrence within 3 years after esophageal preservation was $13.2 \% \quad(95 \% \quad \mathrm{CI}, \quad 4.4 \%-35.5 \%)$, representing 3 of 23 patients, one of whom subsequently died (Figure 2, B). In terms of the site of first recurrence, only one patient presented with an isolated locoregional recurrence that later spread distantly, with

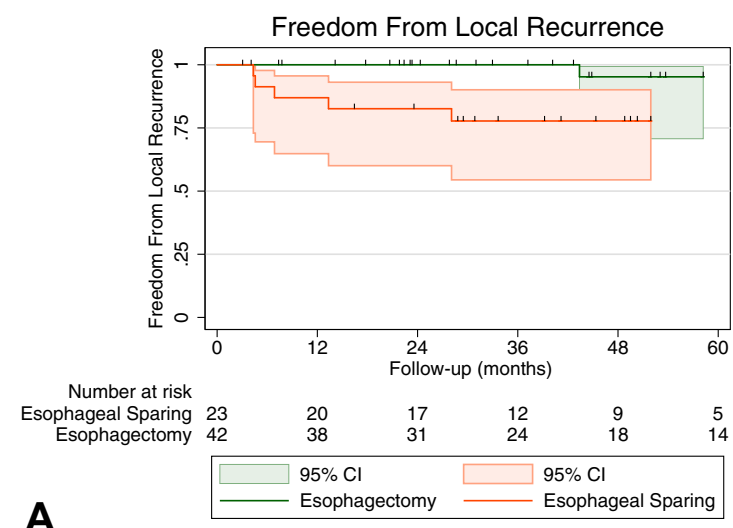

the other 2 patients presenting initially with a distant metastasis.

\section{Recurrence Patterns After Esophagectomy}

Local recurrence within 3 years after esophagectomy was $0 \%$. One patient developed local recurrence at 43 months and died shortly after starting chemotherapy for locally advanced disease.

Regional recurrence within 3 years after esophagectomy was $5.6 \%$ (95\% CI, 1.4\%-20.7\%), which represented 3 of 42 patients. All of these regional recurrences were nonactionable in that they were either detected concurrently with a distant recurrence or after the distant recurrence was discovered. Distant recurrence within 3 years after esophagectomy was $8.1 \%$ (95\% CI, 2.7\%-23.2\%), which

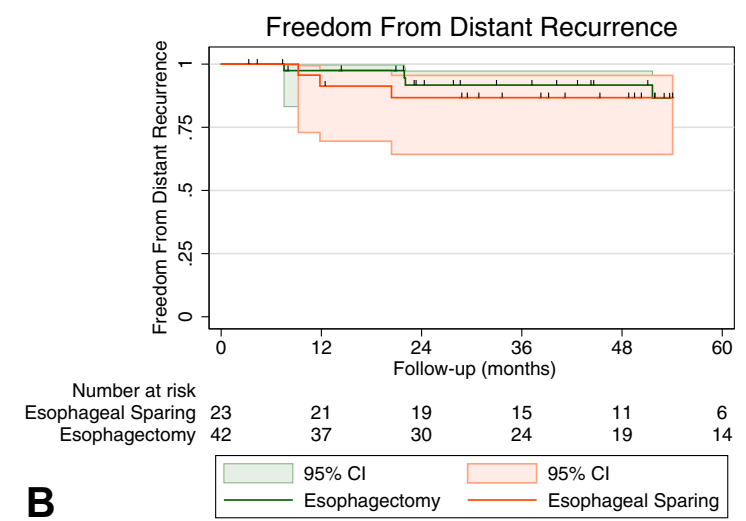

FIGURE 2. Kaplan-Meier freedom from recurrence. A, Freedom from local recurrence $(P=.014)$. B, Freedom from distant recurrence $(P=.440)$. CI, Confidence interval. 
represented 4 of 42 patients. All of the esophagectomy patients with distant recurrence subsequently died.

\section{Risk of Local or Distant Recurrence}

Patients who underwent esophageal preservation had an increased risk of local recurrence (Figure 2, $A ; P=.014$ ). However, there was no difference in the rate of regional recurrence $(P=.685$ ), or distant recurrence (Figure 2, $B$; $P=.440)$ after esophageal preservation.

\section{Stratified Analysis}

All patients were stratified into categories of low, medium, or high risk of nodal metastasis to identify a suspected treatment interaction between local resection approach and risk of nodal metastasis. On the basis of previously published data, we suspected that patients with a low risk of nodal metastasis would fare well with either therapy, but patients with a high risk of nodal metastasis would fare better after esophagectomy.

There were 12 patients with a low risk of lymph node metastasis, 18 with a medium risk, and 35 with a high risk. The median follow-up for low-risk lesions was 47 months, for medium-risk lesions 47 months, and for high-risk lesions 40 months. More patients who underwent esophageal preservation were considered low-risk than those who underwent esophagectomy ( $35 \%$ vs $10 \% ; P=.003)$.

Among the 12 patients with a low risk of nodal metastasis, there were no regional or distant recurrences, and patients fared well regardless of local resection approach. There continued to be no difference among those with a medium risk of lymph node metastases. However, patients who were predicted to have a high risk of nodal metastasis showed a trend toward increased risk of distant recurrence with esophageal preservation (Figure 3, B; $P=.066$ ). High-risk patients were those with LVI or larger, deeper, or poorly differentiated lesions.

\section{Propensity Matched Analysis}

To minimize the risk that our outcomes could be confounded by differences in baseline covariates, patients were matched in a 1:1 fashion on the basis of their propensity to undergo esophageal preservation. Depth of invasion, tumor grade, and presence of LVI were used as covariates to construct the propensity model. Fifteen pairs of matched patients were identified. After propensity matching, patients who underwent esophageal preservation continued to show an increased risk of local recurrence, although this difference was no longer statistically significant (Figure E2; $P=.083$ ). However, there continued to be no difference in the risk of regional or distant recurrence ( $P=.578$ and $P=.658$, respectively).

\section{DISCUSSION}

Therapeutic EMR with esophageal preservation was associated with an increased risk of local recurrence compared with esophagectomy, but no difference in the rate of distant recurrence. A risk-stratified analysis showed that for patients who have a low risk of nodal metastasis, either resection approach resulted in excellent cancer control. However, for patients who harbor a high risk of nodal metastasis, a statistically nonsignificant trend was observed toward an increased risk of distant recurrence after esophageal preservation.

To our knowledge, the largest series on the results of endoscopic resection for submucosal esophageal cancer was reported by Manner et al. ${ }^{13}$ In this study, 66 patients were treated with endoscopic resection, with no tumor-associated deaths, and 5-year survival rate of $84 \%$. This study was limited to low-risk lesions that showed the most favorable likelihood of success: SM1 invasion, macroscopically polypoid or flat, good to moderate differentiation, and no LVI. In contrast, our study included high-risk patients and provides a unique risk stratification. Other centers have reported on their outcomes
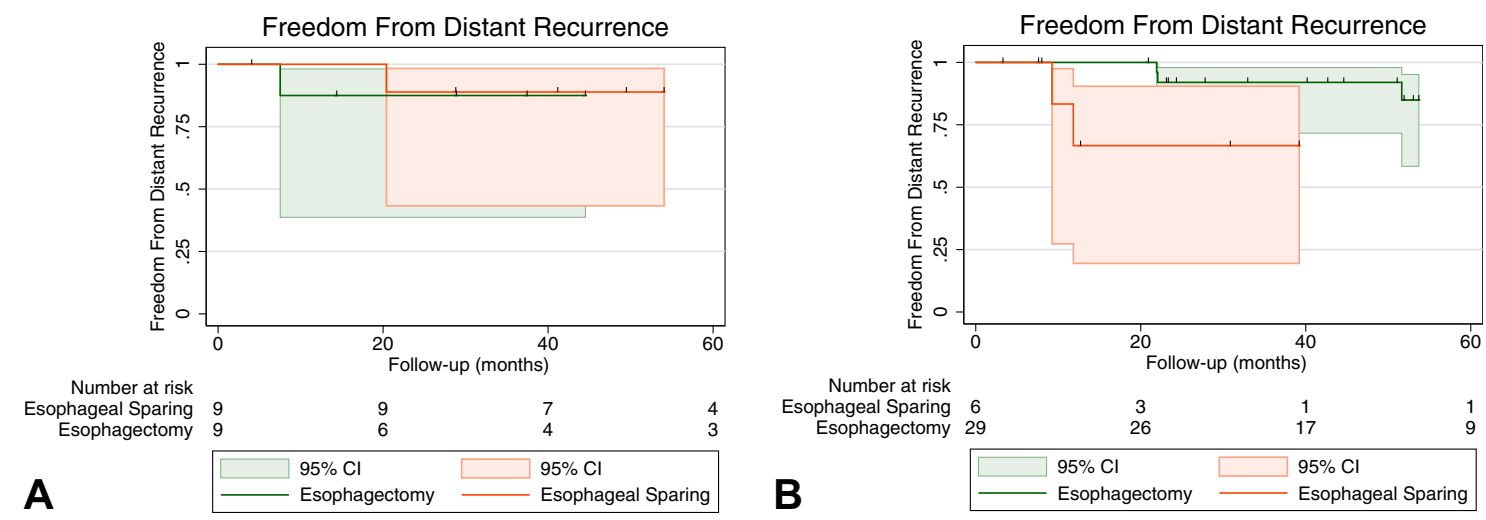

FIGURE 3. Kaplan-Meier freedom from distant recurrence stratified according to risk of lymph node metastasis. A, Freedom from distant recurrence; medium risk $(P=.8532)$. B, Freedom from distant recurrence, high risk $(P=.0660)$. Low risk not pictured because there were no events. $C I$, Confidence interval. 
after endoscopic therapy of submucosal esophageal cancer with broader selection criteria, however, they were all smaller in size with $<30$ patients each and showed no substantial difference from those reported after esophagectomy. ${ }^{15-18}$

Our findings of excellent cancer-related outcomes among patients with a low risk of nodal metastasis mirror these results published by Manner et al. Either local resection strategy for low-risk lesions appears to fare favorably well. Central to this strategy is appropriately defining low-risk lesions, which, in our population included patients with no LVI, well to moderate differentiation, SM1 to SM2 invasion, and size $2 \mathrm{~cm}$ or less. LVI appears to be the most important factor driving the risk of lymph node metastasis, because its hazard ratio is the highest, and statistically LVI has been shown to increase the effect of other variables including depth of invasion. ${ }^{8}$ Indeed, when LVI is not appropriately included as a risk-stratifying variable, SM1 lesions have an unacceptably high risk of lymph node metastasis. ${ }^{6}$ Our findings support previous studies that show that low-risk submucosal esophageal adenocarcinoma may be treated in a fashion similar to mucosal adenocarcinoma.

We expected to find that esophagectomy would lead to improved regional and distant cancer control among high-risk lesions because of the inability of EMR to clear nodal tissue. Although not statistically significant, esophageal preservation showed a trend toward an increased rate of distant recurrence among high-risk lesions. This might be a limitation of the small sample size in our study. It is also important to note that patients who underwent therapeutic EMR might have an attenuated risk of distant recurrence because of the high rate of utilization of adjuvant chemoradiation, as well as the opportunity for salvage therapy after locoregional failures. The presence of these competing explanations makes our analysis a comparative effectiveness study of an esophageal preservation pathway after EMR, because any number of them might lower the rate of distant failure among patients with esophageal preservation. The risk of local failure, or possibly distant failure among high-risk patients, should nonetheless be balanced against the harms of unnecessarily performing an esophagectomy in a patient who did not require one. As mentioned, 11 of 21 esophagectomy patients with a previous diagnostic EMR had no evidence of residual local nor regional disease.

There were several limitations to this study. The first is generalizability. Our data represents experience at a quaternary academic institution, experienced in advanced endoscopy, with a multidisciplinary approach consisting of experienced surgeons, medical oncologists, and radiation oncologists available. There is a risk that these results will not be reproducible at centers that lack substantial multidisciplinary experience. Second, there were important selection effects leading to a bias against the surgical cohort, reflecting higher-risk disease within this cohort.
We attempted to minimize this through careful selection criteria, as well as a secondary analysis using propensity matching. We also implemented a novel scoring system to stratify our results according to the risk of lymph node metastasis. The lack of cross-validation or an independent validation of our scoring system can be overcome with further studies. Last, as mentioned earlier, there is a risk of failing to detect a statistically significant difference.

Despite 1 in 5 submucosal esophageal cancers being treated endoscopically in the United States in 2010, the literature regarding outcomes in this subset of patients is generally weak in quality and needs to be improved. Advancements in biomarker technology might also lead to novel methods of identifying low-risk lesions and requires further study.

\section{CONCLUSIONS}

Esophageal preservation after EMR is a reasonable therapeutic option for patients with submucosal esophageal cancer who harbor low-risk pathologic features, provided that this therapy is given at experienced centers with multidisciplinary care available. Low-risk features include those with no LVI, $2 \mathrm{~cm}$ or less in size, well to moderate differentiation, and within the inner two-thirds of the submucosa. Therapeutic EMR with esophageal preservation led to an increased risk of local recurrence, even despite having preferentially performed esophageal preservation among low-risk patients. However, many of these recurrences were able to be salvaged with either repeat EMR or surgery. Although we were unable to show that patients with high-risk features fare worse after esophageal preservation with EMR, there is strong biologic evidence that the risk for lymph node metastasis is elevated, and our study was not powered for noninferiority. Therefore, we currently recommend esophagectomy for any patients with intermediate- to high-risk features, including those with LVI, poor differentiation, deep invasion, or tumor size larger than $2 \mathrm{~cm}$.

\section{Webcast}

You can watch a Webcast of this AATS meeting presentation by going to: https://aats.blob.core.windows.net/ media/17AM/2017-05-03/RM312/05-03-17_Room312_0754_ Nelson.mp4.

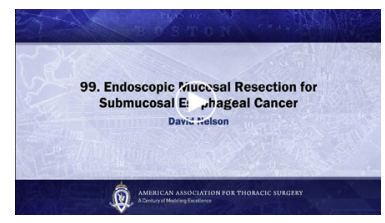

\section{Conflict of Interest Statement}

Authors have nothing to disclose with regard to commercial support. 


\section{References}

1. Siegel RL, Miller KD, Jemal A. Cancer statistics, 2015. CA Cancer J Clin. 2015; 65:5-29.

2. Pech O, Behrens A, May A, Nachbar L, Gossner L, Rabenstein T, et al. Long-term results and risk factor analysis for recurrence after curative endoscopic therapy in 349 patients with high-grade intraepithelial neoplasia and mucosal adenocarcinoma in Barrett's oesophagus. Gut. 2008; 57:1200-6.

3. Pech O, Bollschweiler E, Manner H, Leers J, Ell C, Holscher AH. Comparison between endoscopic and surgical resection of mucosal esophageal adenocarcinoma in Barrett's esophagus at two high-volume centers. Ann Surg. 2011;254:67-72.

4. Nakagawa K, Koike T, Iijima K, Shinkai H, Hatta W, Endo H, et al. Comparison of the long-term outcomes of endoscopic resection for superficial squamous cell carcinoma and adenocarcinoma of the esophagus in Japan. Am J Gastroenterol. 2014;109:348-56.

5. Pech O, May A, Manner H, Behrens A, Pohl J, Weferling M, et al. Long-term efficacy and safety of endoscopic resection for patients with mucosal adenocarcinoma of the esophagus. Gastroenterology. 2014;146:652-60.e1.

6. Sepesi B, Watson TJ, Zhou D, Polomsky M, Litle VR, Jones CE, et al. Are endoscopic therapies appropriate for superficial submucosal esophageal adenocarcinoma? An analysis of esophagectomy specimens. J Am Coll Surg. 2010;210:418-27.

7. Leers JM, DeMeester SR, Oezcelik A, Klipfel N, Ayazi S, Abate E, et al. The prevalence of lymph node metastases in patients with T1 esophageal adenocarcinoma a retrospective review of esophagectomy specimens. Ann Surg. 2011;253:271-8.

8. Lee L, Ronellenfitsch U, Hofstetter WL, Darling G, Gaiser T, Lippert C, et al. Predicting lymph node metastases in early esophageal adenocarcinoma using a simple scoring system. J Am Coll Surg. 2013; 217:191-9.

9. Manner H, Wetzka J, May A, Pauthner M, Pech O, Fisseler-Eckhoff A, et al. Early-stage adenocarcinoma of the esophagus with mid to deep submucosal invasion (pT1b sm2-3): the frequency of lymph-node metastasis depends on macroscopic and histological risk patterns. Dis Esophagus. 2017;30:1-11

10. Ancona E, Rampado S, Cassaro M, Battaglia G, Ruol A, Castoro C, et al. Prediction of lymph node status in superficial esophageal carcinoma. Ann Surg Oncol. 2008; 15:3278-88.

11. Barbour AP, Jones M, Brown I, Gotley DC, Martin I, Thomas J, et al. Risk stratification for early esophageal adenocarcinoma: analysis of lymphatic spread and prognostic factors. Ann Surg Oncol. 2010;17:2494-502.

12. Raja S, Rice TW, Goldblum JR, Rybicki LA, Murthy SC, Mason DP, et al. Esophageal submucosa: the watershed for esophageal cancer. J Thorac Cardiovasc Surg. 2011;142:1403-11.e1.

13. Manner H, Pech O, Heldmann Y, May A, Pohl J, Behrens A, et al. Efficacy, safety, and long-term results of endoscopic treatment for early stage adenocarcinoma of the esophagus with low-risk sm1 invasion. Clin Gastroenterol Hepatol. 2013;11: 630-5. quiz: e45.

14. Merkow RP, Bilimoria KY, Keswani RN, Chung J, Sherman KL, Knab LM, et al. Treatment trends, risk of lymph node metastasis, and outcomes for localized esophageal cancer. J Natl Cancer Inst. 2014;106:dju133.

15. Ballard DD, Choksi N, Lin J, Choi EY, Elmunzer BJ, Appelman H, et al. Outcomes of submucosal (T1b) esophageal adenocarcinomas removed by endoscopic mucosal resection. World J Gastrointest Endosc. 2016;8:763-9.

16. Alvarez Herrero L, Pouw RE, van Vilsteren FGI, ten Kate FJW, Visser M, van Berge Henegouwen MI, et al. Risk of lymph node metastasis associated with deeper invasion by early adenocarcinoma of the esophagus and cardia: study based on endoscopic resection specimens. Endoscopy. 2010; 42:1030-6.

17. Tian J, Prasad GA, Lutzke LS, Lewis JT, Wang KK. Outcomes of T1b esophageal adenocarcinoma patients. Gastrointest Endosc. 2011;74:1201-6.

18. Park HC, Kim DH, Gong EJ, Na HK, Ahn JY, Lee JH, et al. Ten-year experience of esophageal endoscopic submucosal dissection of superficial esophageal neoplasms in a single center. Korean J Intern Med. 2016;31:1064-72.

Key Words: esophageal cancer, EMR, endoscopic mucosal resection, submucosa, surgery, recurrence, adenocarcinoma, endoscopy

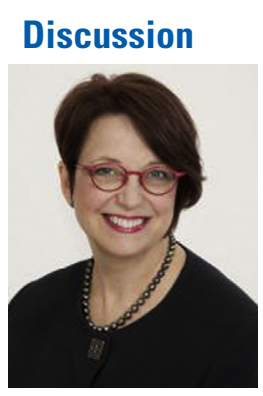

Dr Gail E. Darling (Toronto, Ontario, Canada). First of all, Wayne, I want to congratulate you and your group for this work. Much of the work on these types of problems happens in the GI literature and doesn't come to our forum, and I think it is important for surgeons to be involved in all aspects of care of esophageal cancer patients.

I have a question about the chemoradiation. A lot of your T1b patients didn't get chemoradiation after esophagectomy, and based on your analysis with the high risk, low risk, medium risk, would you now recommend that those patients receive adjuvant therapy?

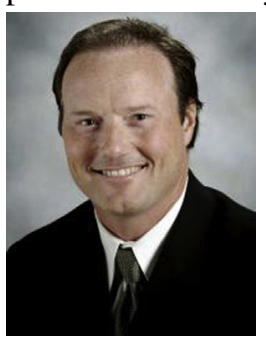

Dr Wayne L. Hofstetter (Houston, $T e x)$. It is a question that is understudied and because the number of patients in the groups is small, we can't make any conclusions here. We have been proposing to study this group of patients. In fact, we ran a nonrandomized prospective trial on $\mathrm{T} 1 \mathrm{~b}$ patients enrolling them to multimodality therapy, but we could only accrue 3 patients to that study over a period of about a year and it ultimately closed from lack of patients.

I think that based on the data that has been published all of us acknowledge that patients who have pT1b disease are not necessarily performing as if they had early disease. These patients have systemic disease in a significant number of cases due to invasion in the lymphovascular space in the submucosa. The question is whether or not the addition of chemoradiation affects that systemic disease, and as you saw in the high-risk group, the failures are distant. So I don't actually know the answer to your question. I think it is a great question, should we be offering chemoradiation to patients who have LVI? The other question is, is it going to be effective if we do give it? There isn't any good data out there, it is something we should probably answer in a collaborative setting.

Dr Darling. Some of the patients had esophagectomy upfront without having an EMR first, and how was that decision made? Why didn't they all have EMR first and then go to either sparing or esophagectomy?

Dr Hofstetter. There has been an evolution. I started doing EMRs in 2006 and for a number of years I was only the one doing the EMRs up until about a year ago when the GI guys realized that, hey, this is something that was kind of fun that they wanted to do it too. But I think that in that transition we didn't recognize that pathologic T1b came out of a lot of different clinical stages. And as you all recognize from other work, clinical T2 about $30 \%$ of the time is actually pathologic $\mathrm{T} 1$, and so early in our experience those 
patients were never referred over for an EMR. So we encourage referral for diagnostic EMR when you have a tumor that is $2 \mathrm{~cm}$ or smaller in size, and is not very positron emission tomography avid and occupies less than 50\% circumference of the esophagus. This is regardless of what the endoscopist says the endoscopic ultrasound (EUS) depth is. If the tumor looks like it could be taken out by EMR, then a diagnostic EMR is worth a shot.

So there were 2 things. Number one, there has to be buyin from your entire surgical group that if the tumor looks amenable to send the patient over for a diagnostic EMR to get further information about it. That resection will give more information about LVI and potential risk of having nodal disease than can be obtained from a biopsy alone and it is more accurate than EUS in determination of depth of invasion. And number 2, is not completely trusting what the endoscopist is telling you about the EUS. So I think more and more probably the central message is to try an EMR if you think you can.

Dr Darling. I am curious about the role of surgeons versus gastroenterologists. I suspect in many of our institutions all this work, the initial diagnosis, the EUS, the EMR, is done by our gastroenterologists rather than by the surgeons. What would you advise us as esophageal surgeons? How should we interact with the GI group? Should we try and take that component of the practice back, do our own EUS, EMR?

Dr Hofstetter. Everyone has to arrange these politics in different ways. I was able to arrange it so that the GI group was doing EUS and ablation so there was plenty of esophageal work for them to do on the same patients, but I wanted to do the initial resections and follow-up. That has become a nice symbiotic relationship for us, but I admit that this may not be possible in every hospital setting. Barring the ability for the surgeon to perform the EMRs, I think that a multidisciplinary conference is absolutely critical. We had to start serving lunch for the first 5 years to get everybody on board, get everybody in the room, and then once everyone got the idea that we were all going to present all of the cases, not just the tough cases but all cases, bring everything out in the light of day and let the sun shine on it, that helped out quite a bit.

Dr Darling. Excellent work.

Dr Hofstetter. Thanks, Gail. I appreciate your discussion.

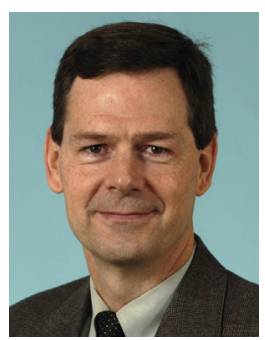

Dr Bryan Fitch Meyers (St Louis, $M o$ ). That is great work you have accomplished, and I am sorry that the gastroenterologists have discovered how fun and easy it is and they are now kind of competing with you.

I noticed since the abstract your overall numbers went up about $15 \%$ but then the numbers of the organ preservation went down. You mentioned that in the talk but it is worth elaborating a little bit more as to what led to those people changing their mind or changing their ultimate therapy?

Dr Hofstetter. You are correct that our abstract was a little bit different. This is because as Dr Nelson initially began to crunch the numbers, he felt like it was very important for us to include all patients including those resected with a positive deep margin, but this ultimately became a very confusing subset of patients. There were 10 patients with a positive deep margin. These are patients that I would never have considered potentially cured with local therapy. Subsequently it became clear that the management of these patients was so different that we were forced to exclude these; so the numbers did change.

It is important to recognize that the patient populations that we are discussing are selected subgroups. This is similarly true for previous work that described this subset of pT1b patients such as the Manner study out of Germany. However, our study population was unique in that we included the low risk group and also the intermediate to high risk groups. The Manner study, which included a hundred or so patients, would all fall into a low risk group. So we did want to include all of the completely resected patients to make this a realistic depiction of what was happening in the clinic setting. I hope that my long-winded response answers your question.

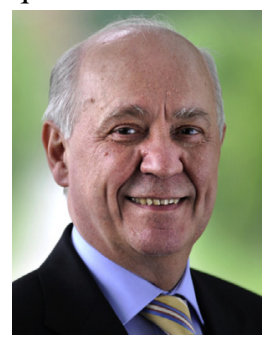

Dr Toni Lerut (Leuven, Belgium). You identified the size of the tumor as a risk factor, but, as you know, there is also a very high interest in looking into the depth of infiltration, SM1, SM2, SM3. Are your pathologists looking at this?

Dr Hofstetter. Based on your question and the reviewer's responses we have requested our pathologist to comment on the submucosal depth of invasion and we have included those results in the study.

I am not convinced that submucosal depth is the absolute indicator of whether or not the patient is going to succeed after an EMR. I am more convinced that other factors such as size and LVI are the most important predictors. There is a somewhat subjective determination of submucosal depth differentiation (SM1-3) and in our experience also a lack of agreement among expert pathologists.

Dr Lerut. What I can comment on is that when it is clinically N0 and it is an SM1, you have a 10\% chance of positive lymph nodes, if you go to SM2 it's $50 \%$, for SM3, even more in our experience. 


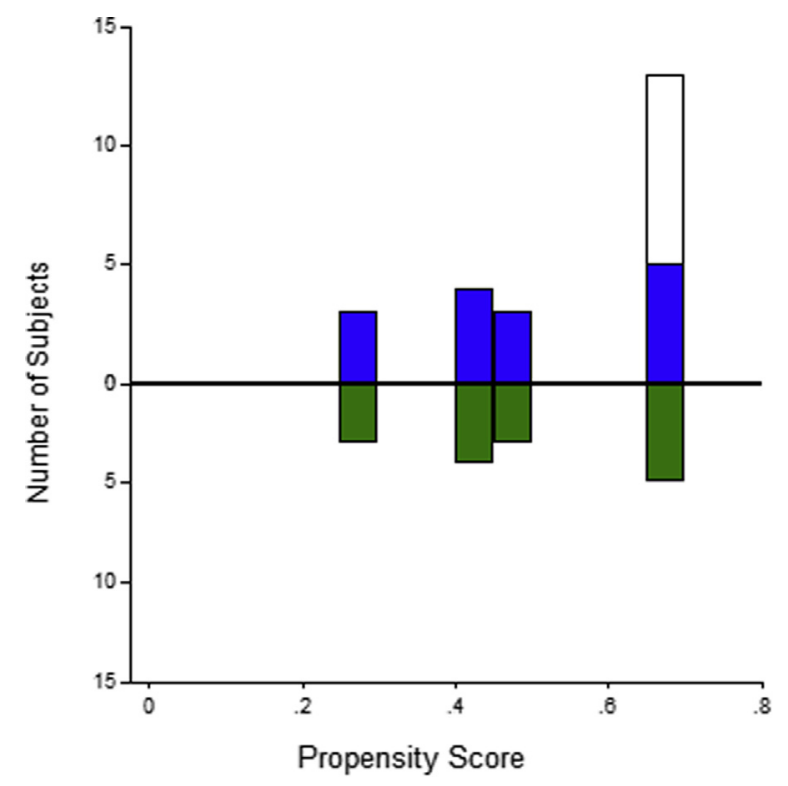

FIGURE E1. Mirror histograms. 


\section{Unmatched}

Freedom From Local Recurrence $(P=.014)$

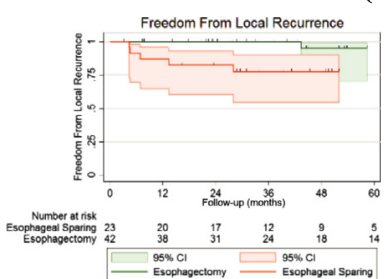

Matched

Freedom From Local Recurrence $(P=.083)$

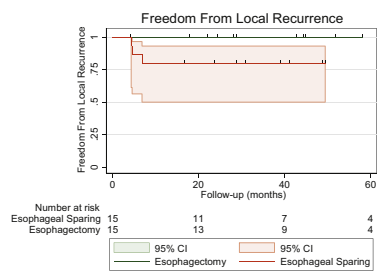

Freedom From Regional Recurrence $(P=$. 685 $)$

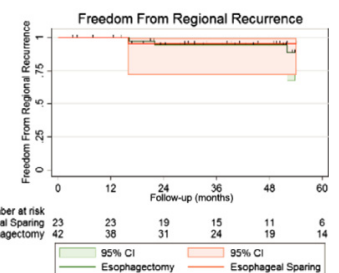

Freedom From Regional Recurrence $(P=.578)$

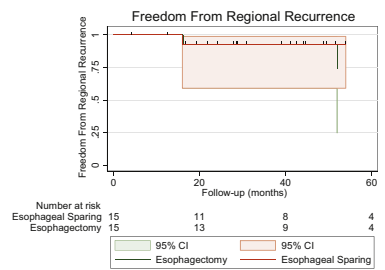

Freedom From Distant Recurrence $(P=.440)$

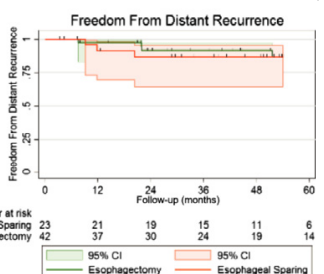

Freedom From Distant Recurrence $(P=.658)$

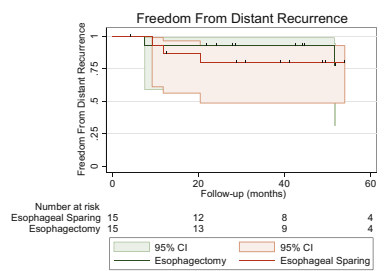

FIGURE E2. Kaplan-Meier freedom from recurrence before and after propensity matching. $C I$, Confidence interval. 
TABLE E1. Baseline demographic and tumor characteristics among propensity-matched cohort

\begin{tabular}{|c|c|c|c|}
\hline & $\begin{array}{c}\text { Esophageal preservation } \\
\qquad(\mathbf{n}=15)\end{array}$ & Esophagectomy $(n=15)$ & $P$ value \\
\hline Median age, y & 72.2 (IQR, 63.8-76.3) & 65.0 (IQR, 60.0-72.0) & .152 \\
\hline Median follow-up, mo & 40.9 & 44.3 & .694 \\
\hline \multicolumn{4}{|l|}{ Sex } \\
\hline Male & $13(87 \%)$ & $14(93 \%)$ & \\
\hline Female & $2(13 \%)$ & $1(7 \%)$ & 1.000 \\
\hline Long Barrett $(>3 \mathrm{~cm})$ & $8(53 \%)$ & $6(40 \%)$ & .538 \\
\hline \multicolumn{4}{|l|}{ Depth of invasion } \\
\hline SM1 & $13(87 \%)$ & $9(60 \%)$ & \\
\hline SM2 & $2(13 \%)$ & $6(40 \%)$ & \\
\hline SM3 & 0 & 0 & .215 \\
\hline \multicolumn{4}{|l|}{ Grade } \\
\hline Moderate & $8(53 \%)$ & $7(47 \%)$ & \\
\hline Poor & $7(47 \%)$ & $8(53 \%)$ & .715 \\
\hline \multicolumn{4}{|l|}{ Tumor location } \\
\hline Distal & $5(33 \%)$ & $6(40 \%)$ & \\
\hline GEJ & $10(67 \%)$ & $9(60 \%)$ & .705 \\
\hline Endoscopic tumor size, $\mathrm{cm}$ & 1.5 (IQR, 0.6-2.0) & 2 (IQR, 1.0-3.0) & .130 \\
\hline Lymphovascular invasion & $6(40 \%)$ & $5(33 \%)$ & .705 \\
\hline
\end{tabular}

$I Q R$, Interquartile range; $S M$, submucosal layer; $G E J$, gastroesophageal junction. 